\title{
Time-to-effect guided pulmonary vein isolation utilizing the third-generation versus second generation cryoballoon: One year clinical success
}

Christian-Hendrik Heeger ${ }^{1,2,3}$, Christopher Schuette ${ }^{2}$, Valentina Seitelberger ${ }^{2}$, Erik Wissner ${ }^{4}$, Andreas Rillig ${ }^{2,5}$, Shibu Mathew ${ }^{2}$, Bruno Reissmann ${ }^{2}$, Christine Lemes ${ }^{2}$, Tilman Maurer ${ }^{2}$, Thomas Fink ${ }^{1,2,3}$, Osamu Inaba ${ }^{2}$,

Naotaka Hashiguchi ${ }^{2}$, Francesco Santoro ${ }^{2}$, Feifan Ouyang ${ }^{2,6}$, Karl-Heinz Kuck ${ }^{2}$, Andreas Metzner ${ }^{2,5}$

${ }^{1}$ University Heart Center Luebeck, Medical Clinic II (Department of Cardiology, Angiology and Intensive Care Medicine), Sektion Elektropjysiologie, University Hospital Schleswig-Holstein, Germany

${ }^{2}$ Department of Cardiology, Asklepios Klinik St. Georg, Hamburg, Germany

${ }^{3}$ DZHK (German Center for Cardiovascular Research), Partner Site Hamburg/Kiel/Lübeck, Berlin, Germany

${ }^{4}$ Division of Cardiology, University of Illinois at Chicago, United States

${ }^{5}$ Department of Cardiology-Electrophysiology, University Hospital Hamburg,

University Heart Center Hamburg, Hamburg, Germany

${ }^{6}$ Fuwai Hospital / National Center of Cardiovascular Diseases, Beijing, China

\begin{abstract}
Background: The second-generation cryoballoon (CB2) provides effective and durable pulmonary vein isolation (PVI) associated with encouraging and reproducible clinical outcome data. The latest-generation cryoballoon (CB3) incorporates a $40 \%$ shorter distal tip, thus allowing for an increased rate of PVI real-time signal recording and facilitating individualized ablation strategies taking the time-to-effect (TTE) into account. However, whether this characteristic translates into favorable clinical success has not been evaluated yet. Herein was investigated 1-year clinical success after CB3 in comparison to CB2 based-PVI.

Methods: One hundred and ten consecutive patients with paroxysmal or short-standing persistent atrial fibrillation $(A F)$ underwent $C B 2(n=55$ patients $)$-or CB3 ( $n=55$ patients $)$-based PVI. The freeze-cycle duration was set to TTE $+120 \mathrm{~s}$ if TTE could be recorded, otherwise a fixed freeze-cycle duration of $180 \mathrm{~s}$ was applied.

Results: A total of 217/218 (99\%, CB3) and 217/217 (100\%, CB2) pulmonary veins (PV) were successfully isolated. The real-time PVI visualization rate was $69.2 \%$ (CB3) and $54.8 \%$ (CB2; $p=0.0392)$. The mean freeze-cycle duration was $194 \pm 77$ s (CB3) and $206 \pm 85 s(C B 2 ; p=0.132)$, respectively. During a median follow-up of 409 days (interquartile range [IQR] 378-421, CB3) and 432 days (IQR 394-455, CB2) $73.6 \%$ (CB3) and $73.1 \%$ of patients (CB2) remained in stable sinus rhythm after a single procedure $(p=0.806)$.

Conclusions: A higher rate of real-time electrical $P V$ recordings was seen using the CB3 as compared to CB2. There was no difference in 1-year clinical follow-up. (Cardiol J 2019; 26, 4: 368-374)

Key words: atrial fibrillation, pulmonary vein isolation, cryoballoon, long-term outcome
\end{abstract}

Address for correspondence: Christian-Hendrik Heeger, MD, University Heart Center Luebeck, Medical Clinic II (Department of Cardiology, Angiology and Intensive Care Medicine), Division of Elektropysiology, University Hospital Schleswig-Holstein, Germany, tel: ++49-(0)451-500 44551, fax: ++49-(0)451-500 44585, e-mail: christian.heeger@gmx.net Received: 13.02 .2018 Accepted: 2.04 .2018 


\section{Introduction}

The second-generation cryoballoon (CB2, Arctic Front Advance, Medtronic, Inc., Minneapolis, MN, USA) for pulmonary vein isolation (PVI) has demonstrated high procedural success rates and convincing clinical outcome data for patients with paroxysmal (PAF) and persistent atrial fibrillation (persAF) [1-4]. The "Fire And Ice" trial proved non-inferiority of CB2- to radiofrequency (RF)-based PVI with regard to efficacy and safety for treatment of patients with PAF [5]. In consequence the latest $\mathrm{AF}$ guidelines state that PVI should be performed using either RF or CB catheters [6]. Current ablation strategies predominantly apply fixed freeze-cycle durations of 180-240 s, oftentimes followed by a bonus-freeze cycle. Recently protocols were modified aiming for shorter and fewer CB-applications and demonstrated comparable clinical outcomes [7, 8]. After identifying the time to pulmonary vein (PV) isolation (time-to-effect [TTE]) as an essential indicator for durable PVI latest ablation strategies have been implemented the individual TTE [9]. The long distal tip of the CB2 limits proper visualization of PV since it prevents proximal positioning of the circular mapping catheter (Achieve, Medtronic) in close proximity to the CB's surface. The third-generation cryoballoon (CB3, Arctic Front Advance ST, Medtronic) incorporates a $40 \%$ shorter tip facilitating a more proximal Achieve position. As a consequence initial studies found a higher rate of real-time PV recordings when utilizing the CB3 compared to CB2 [9-11]. However, whether this characteristic translates into favorable 1-year clinical success has not been evaluated yet. Herein was investigated the 1-year clinical outcomes after CB3 in comparison to CB2 based-PVI.

\section{Methods}

\section{Inclusion and exclusion criteria}

Consecutive patients with symptomatic, drugrefractory PAF or short standing PersAF (duration $\leq 3$ months) consented to $\mathrm{CB}$-based PVI and were treated with second-generation cryoballoon (CB2 group, control), followed by 55 consecutively treated patients with the third-generation cryoballoon (CB3 group). Data on acute success of a limited number of patients was published before [9]. Exclusion criteria were prior left atrial (LA) ablation attempts, a LA diameter $>60 \mathrm{~mm}$, severe valvular heart disease or contraindications to postinterventional oral anticoagulation. Transesophageal echo- cardiography was performed in all patients prior to PVI to rule out intracardiac thrombi and to assess LA diameter. No further pre-procedural imaging was performed. The procedure was performed in patients on vitamin $\mathrm{K}$ antagonists under therapeutic international normalized ratio (INR) values of $2-3$. New oral anticoagulants were stopped 1 day prior to the procedure and continued $6 \mathrm{~h}$ post ablation. All patients gave written informed consent and all patient information was anonymized. The study was approved by the local ethic's board (ethical review board number: WF-028/15) and was performed in accordance with ethical standards laid down in the 1964 Declaration of Helsinki and its later amendments.

\section{Intraprocedural management}

The intraprocedural management has been described in detail in previous studies by the present group [4, 9, 12]. In brief, the procedure was performed under deep sedation using midazolam, sufentanyl and propofol. Prior to transseptal puncture one diagnostic catheter was introduced via the right femoral vein and positioned within the coronary sinus (7 F, Webster TM, Biosense Webster, Inc. Diamond Bar, CA, USA). Single transseptal puncture was performed under fluoroscopic guidance using a modified Brockenbrough technique and an $8.5 \mathrm{~F}$ transseptal sheath (SL1, St. Jude Medical, Inc., St. Paul, MN, USA). Selective PV angiography was performed to identify the PV ostia. The transseptal sheath was exchanged over a guidewire for a $12 \mathrm{~F}$ steerable sheath (Flexcath Advance, Medtronic). After transseptal puncture heparin boluses were administered targeting an activated clotting time of $>300 \mathrm{~s}$.

\section{Cryoballoon-based PVI}

The $28 \mathrm{~mm} \mathrm{CB}$ was advanced into the LA via the $12 \mathrm{~F}$ steerable sheath using the Achieve catheter (20 mm diameter, Medtronic, Inc.) for guidance. The $\mathrm{CB}$ was inflated proximal to the $\mathrm{PV}$ ostium followed by gentle push aiming for complete $\mathrm{PV}$ sealing. Occlusion of the PV ostium was verified by contrast dye injections. Standard freeze-cycle duration was TTE +120 s. If the TTE could not be visualized, the freeze-cycle duration was set to $180 \mathrm{~s}$. No additional bonus freeze-cycle was applied. The procedural endpoint was defined as persistent PVI verified by Achieve catheter (Medtronic, Inc.) recordings after the last $\mathrm{CB}$ application.

A spiral temperature probe (Sensitherm, St. Jude Medical) was advanced into the esophagus to monitor esophageal temperatures during each 
energy application. The intraluminal esophageal temperature cut-off was set at $15^{\circ} \mathrm{C}$. During energy delivery along the septal $\mathrm{PVs}$, continuous phrenic nerve $(\mathrm{PN})$ pacing was performed using a diagnostic catheter introduced into the superior vena cava (7 F, Webster TM, Biosense Webster, Inc.). Pacing was set at maximum output and pulse width $(12 \mathrm{~mA}, 2.9 \mathrm{~ms})$ and a cycle length of 1200 ms. PN capture was monitored by tactile feedback of diaphragmatic contraction and assessment of the right diaphragmatic compound motor action potential (CMAP). Energy delivery was interrupted immediately if weakening or loss of diaphragmatic contraction was noted or a decrease of CMAP amplitude of $\geq 30 \%$ was seen. In case of persistent PN palsy, no further cryoenergy was delivered to septal PVs.

\section{Postprocedural care}

Following ablation, all patients underwent transthoracic echocardiography to rule out a pericardial effusion. Low molecular-weight heparin was administered in patients on vitamin $\mathrm{K}$ antagonists and an INR $<2.0$ until a therapeutic INR of $2-3$ was achieved. New oral anticoagulants were re-initiated $6 \mathrm{~h}$ post ablation. Anticoagulation was continued for at least 3 months and continued thereafter based on the individual $\mathrm{CHA}_{2} \mathrm{DS}_{2}$-VASc score. Previously ineffective antiarrhythmic drugs were continued for 3 months post ablation [4, 12].

All patients were treated with proton-pump inhibitors for 6 weeks.

\section{Clinical follow-up and study endpoints}

Following a blanking period of 3 months, patients completed outpatient clinic visits at 3,6 and 12 months and thereafter in 6 -month intervals which included electrocardiograms (ECGs) and 24 h-Holter ECGs. In addition, regular telephonic interviews were performed. Additional outpatient clinic visits were immediately initiated in case of symptoms suggestive of recurrent arrhythmia. The primary endpoint was defined as recurrence of any symptomatic or documented $\mathrm{AF}$ episode $>30 \mathrm{~s}$ following a blanking period of 3 months. Secondary endpoints were acute procedural data and procedure related complications such as phrenic nerve palsy, cerebral embolism, pericardial effusion/tamponade or atrioesophageal fistula.

\section{Statistical analysis}

Differences in metric variables between the two groups were analyzed with t-test if data were normally distributed, and otherwise with Wilcoxon-
-Mann-Whitney test. Differences between categorical variables were evaluated using $\chi^{2}$ test or the Fisher exact test in case of small expected cell frequencies. PVI data was analyzed using mixed models. Linear mixed models were used for continuous data. Generalized linear mixed models were used for binary or count data. A hierarchical logistic regression model was applied for binary data. A Poisson distribution was assumed for count data. All p-values are two-sided and a p-value $<0.05$ was considered significant. All calculations were performed with statistical analysis software SAS (SAS Institute Inc., version 9.3, Cary, NC, USA) [12].

\section{Results}

\section{Patient characteristics}

A total of 110 consecutive patients underwent $28 \mathrm{~mm}$ CB-based PVI utilizing the CB2 $(\mathrm{n}=55)$ and CB3 ( $\mathrm{n}=55)$ (Fig. 1). Patient baseline characteristics are depicted in Table 1. No differences were identified between the groups.

\section{Acute ablation results}

Acute ablation results are depicted in Table 2. In 110 patients, a total of $435 \mathrm{PVs}$ were identified and targeted. One right superior pulmonary vein (CB3 group) was not isolated due to phrenic nerve palsy during $\mathrm{CB}$ application along the ipsilateral right inferior pulmonary vein. All other PVs were successfully isolated $(434 / 435,99.8 \%)$. Real-time PVI was visualized in $69.2 \%$ of patients in the CB3 and in $54.8 \%$ of patients in the CB2 group, respectively $(\mathrm{p}=0.0392)$. A significant difference regarding the mean minimal $\mathrm{CB}$ temperatures was assessed $\left(-45^{\circ} \mathrm{C}, \mathrm{CB} 3\right.$ and $\left.-48^{\circ} \mathrm{C}, \mathrm{CB} 2 ; \mathrm{p}<0.0001\right)$. Yet, no difference was found for the mean total number of $\mathrm{CB}$ freeze-cycles per PV until PVI, the incidence of PVI after first CB freeze-cycle, the mean TTE, total freeze-time applied, mean CB temperature after $30 \mathrm{~s}$ of the freeze-cycle, or mean minimal esophageal temperature. Furthermore, no differences were found for total procedure time, total fluoroscopy time, and total amount of contrast dye (Table 2).

All procedures were performed by three highly experienced operators and no differences were observed with regard to catheter maneuverability and stability.

\section{Peri- and postprocedural complications}

Transient PN palsy occurred in 1/55 (1.8\%) patients in the $\mathrm{CB} 3$ group during energy delivery along the RIPV with full recovery of nerve function 


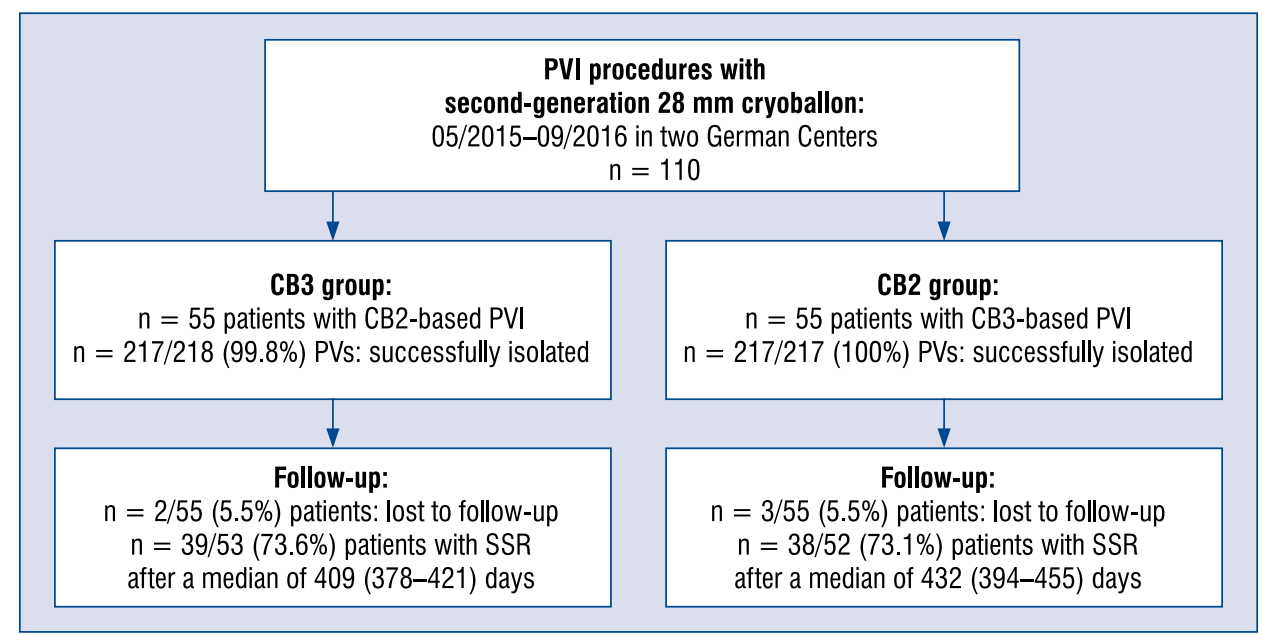

Figure 1. Flow-chart; $\mathrm{AF}$ - atrial fibrillation; PVI — pulmonary vein isolation; SSR — stable sinus rhythm.

Table 1. Baseline characteristics

\begin{tabular}{lccc}
\hline Variable & All & CB3 & CB2 \\
\hline Patients & 110 & 55 & 55 \\
Age $[$ years] & $62.1 \pm 11.4$ & $60.4 \pm 11.1$ & $63.8 \pm 11.5$ \\
Body mass index $\left[\mathrm{kg} / \mathrm{m}^{2}\right]$ & $27.5 \pm 4.87$ & $28.3 \pm 4.9$ & $26.7 \pm 4.7$ \\
Female gender & $33(70 \%)$ & $16(70.9 \%)$ & $17(69.1 \%)$ \\
Paroxysmal AF & $71(64.6 \%)$ & $34(61.8 \%)$ & $37(67.3 \%)$ \\
Short persistent AF & $39(34.5)$ & $21(38.2)$ & $18(32.7)$ \\
Duration of AF [months] & $24(12,48)$ & $24(14,48)$ & $16.5(7.5,48)$ \\
LA-size [mm] & $45.0 \pm 6$ & $45.9 \pm 7$ & $44.1 \pm 5.7$ \\
Congestive heart failure & $12(11 \%)$ & $8(6 \%)$ & $4(7 \%)$ \\
Arterial hypertension & $69(63 \%)$ & $35(64 \%)$ & $34(62 \%)$ \\
Diabetes mellitus type 2 & $12(11 \%)$ & $9(16 \%)$ & $3(5.5 \%)$ \\
Previous TIA/stroke & $7(6 \%)$ & $3(5 \%)$ & $4(7 \%)$ \\
Coronary artery disease & $12(11 \%)$ & $4(7 \%)$ & $8(6 \%)$ \\
CHA $_{2}$ DS $-V A S c$ score & $2.0(1.0,2.0)$ & $2.0(1.0,2.0)$ & $2.0(1.0,2.0)$ \\
EHRA classification & $2.0(2.0,3.0)$ & $2.0(2.0,3.0)$ & $2.0(2.0,3.0)$ \\
\hline
\end{tabular}

Values expressed as number (percentage), mean \pm standard deviation or median $\left(25^{\text {th }}, 75^{\text {th }}\right.$ percentiles) as appropriate. Test of no regression found no differences between the groups $(p=0.389)$. AF — atrial fibrillation; CB2 - second-generation cryoballoon; CB3 - third-generation cryoballoon; EHRA - European Heart Rhythm Association; LA — left atrium; TIA — transient ischemic attack

after 15 min. No pericardial effusion, pericardial tamponade, symptomatic PV stenosis, transient ischemic attack/stroke, or atrioesophageal fistula were observed in any group.

\section{Clinical follow-up}

Clinical 1-year follow-up was obtained in 105/110 (95.5\%) patients, while 5/110 (4.5\%) patients were lost to follow-up. During a median follow-up of 409 (interquartile range [IQR] 378-421, CB3) days and 432 days (IQR 394-455 , CB2) $73.6 \%$ of patients (CB3) and $73.1 \%$ of patients (CB2) remained in stable sinus rhythm ( $\mathrm{p}=0.806$ ) following a single $28 \mathrm{~mm} \mathrm{CB}$-based PVI procedure including a 3-month blanking period (Fig. 2). 
Table 2. Procedural details.

\begin{tabular}{lcccc}
\hline Variable & All & CB3 & CB2 & p \\
\hline Number of PVs & 435 & 218 & 217 \\
Total CB cycles until PVI & $1.1 \pm 0.5$ & $1.1 \pm 0.6$ & $1.1 \pm 0.4$ & 0.128 \\
Number of isolated PVs & $434 / 435(99 \%)$ & $217 / 218(99 \%)$ & $217 / 217(100 \%)$ & 0.316 \\
Number of isolated PVs after $1^{\text {st }}$ cycle & $390 / 435(89.7 \%)$ & $191 / 218(88 \%)$ & $199 / 217(92 \%)$ & 1.000 \\
Minimal temperature $\left[{ }^{\circ} \mathrm{C}\right]$ & $-47 \pm 6$ & $-45 \pm 6$ & $-48 \pm 6$ & $<0.0001$ \\
Minimal esophageal-temperature $\left[{ }^{\circ} \mathrm{C}\right]$ & $33.7 \pm 4$ & $33.9 \pm 4$ & $33.5 \pm 4$ & 0.474 \\
Real-time recordings & $270(62.1 \%)$ & $151(69.2 \%)$ & $119(54.8 \%)$ & 0.0392 \\
Time to PVI $[\mathrm{s}]$ & $47 \pm 32$ & $48 \pm 32$ & $45 \pm 32$ & 1.000 \\
Temperature after $30 \mathrm{~s} \mathrm{CB} \mathrm{cycle} \mathrm{duration}\left[{ }^{\circ} \mathrm{C}\right]$ & $-31 \pm 10$ & $-29 \pm 11$ & $-32 \pm 9$ & 0.075 \\
Duration of total freezing time $[\mathrm{s}]$ & $200 \pm 81$ & $194 \pm 77$ & $206 \pm 85$ & 0.132 \\
Total procedure time $[\mathrm{min}]$ & $92 \pm 28$ & $94 \pm 30$ & $90 \pm 27$ & 0.525 \\
Total flouroscopy time $[\mathrm{min}]$ & $17(13,23)$ & $19(14,27)$ & $16(13,20)$ & 0.389 \\
Total amount of contrast $[\mathrm{mL}]$ & $115 \pm 25$ & $115 \pm 25$ & $116 \pm 25$ & 0.792 \\
\hline
\end{tabular}

Values expressed as number (percentage), mean \pm standard deviation or median $\left(25^{\text {th }}, 75^{\text {th }}\right.$ percentiles) as appropriate. CB -28 mm cryoballoon; $\mathrm{CB} 2$ - second-generation cryoballoon; CB3 - third-generation cryoballoon; PV(s) - pulmonary vein(s); PVI — pulmonary vein isolation



Figure 2. Recurrence free survival between the groups ( $\mathrm{n}=110$ patients). Kaplan-Meier curve demonstrating the relative proportion of patients in stable sinus rhythm following initial pulmonary vein isolation (PVI) using the third-generation cryoballoon (CB3) compared to the second-generation cryoballoon (CB2) including a 3-month blanking period. During a median follow-up of 409 (interquartile range [IQR] 378-421, CB3) days and 432 days (IQR 394-455, CB2) $73.6 \%$ of patients (CB3) and $73.1 \%$ of patients (CB2) remained in stable sinus rhythm without any differences between the groups ( $p=0.806$ ) following a single $28 \mathrm{~mm}$ CB-based PVI procedure including a 3-month blanking period. No significant difference was found for the follow-up duration $(p=0.071)$.

\section{Discussion}

The current study set out to compare procedural characteristics and acute and 1-year clinical efficacy of the novel $28 \mathrm{~mm}$ CB3 to the CB2 for PVI in patients with $\mathrm{PAF}$ and short-standing persAF. The major findings are 1) the CB3 provides an identical rate of acute PVI during the index freeze-cycle as the CB2, 2) the incidence of PVs with real-time signal recordings was significantly higher in the CB3 group, 3) procedure and fluoroscopy times were not different although a trend towards shorter freeze-cycles was observed in the CB3 group when considering the individual TTE, and 4) there were no statistical significant differences in the 1-year clinical outcomes between both groups.

Recent studies focusing on CB2 ablation demonstrated a high rate of PVI (84-90\%) during the index freeze cycle [12-14], superior 1- and 2-year clinical outcomes as compared to first-generation CB even if "no-bonus" freeze protocols or a shorter freeze-cycle durations were applied, and a high 
rate of durable PVI as demonstrated in patients undergoing repeat procedures for recurrent atrial tachyarrhythmias after previous second-generation $\mathrm{CB}$ ablation [15]. Modified ablation strategies taking the individual TTE into account are currently under investigation. An essential prerequisite for TTE-based ablation protocols is a considerably high incidence of real-time PV potential recordings. Incorporating individual TTE into ablation protocols may reduce total energy transfer per PV which in turn may contribute to an even better safety-profile of CB-based PVI [16].

The current study confirms that a high rate of PVI with initial freeze-cycle can be achieved with both, the CB3 and CB2 (86\% vs. $92 \%$, $\mathrm{p}=0.14$ ). Although mean minimal balloon-temperature measured when applying CB2 was significantly lower than with CB3, acute efficacy was similar for both $\mathrm{CB}$ generations. Observed differences in minimal balloon-temperatures might be caused by a slightly changed position of the temperature probe of CB3 to a more proximal position compared to CB2. This fact causes a minimally longer distance of the returning gas to the temperature probe and therefore higher returning gas temperatures. Of note, no significant difference was assessed between both groups for balloon temperature reached at $30 \mathrm{~s}$ of the respective freeze-cycle [17]. There was also no difference when comparing the lowest intraluminal esophageal temperatures. These facts additionally support the observation of an equal acute efficacy of both CB-generations.

Characteristic complications of CB-based PVI such as $\mathrm{PN}$ palsy or a significant drop in intraluminal esophageal temperatures potentially resulting in esophageal thermal injury typically occur at later stages of the freeze-cycles [18]. Therefore, recent ablation protocols aim not only to reduce of the number of freeze-cycles per PV but especially for shorter freeze-cycle durations to further improve the safety profile of CB-based PVI [17]. The Achieve catheter provides online visualization of $\mathrm{PV}$ signals. However, the relatively long distal tip of CB2 may prevent proper positioning of the Achieve catheter at proximal PV ostium, thus limiting real-time signal recording in a considerable number of PVs and individualized ablation protocols considering TTE $[14,19]$. The novel CB3 was modified and incorporates a $40 \%$ shorter distal tip, potentially facilitating more proximal positioning of Achieve-catheter and increased rates of real-time PV signal recordings. Accordingly, the current study found a significantly higher percentage of real-time PVI recordings for CB3 as compared to CB2 group (69.2\% vs. $54.8 \%$, $\mathrm{p}=0.0392$ ) and in consequence allowed for a higher percentage of PVs in which individual TTE was taken into account without affecting overall clinical outcomes. In conclusion, this means that a shorter balloon tip will further facilitate individualized ablation strategies and might further improve the safety profile of $\mathrm{CB}$ ablation due to shorter but efficient energy transfer [20]. Although one group reported on balloon dislodgement during hockey stick maneuvers caused by the shortened distal tip of CB3 [21], no differences concerning catheter stability and catheter maneuverability have been observed in the present analysis. Although CB3 was taken from the market it would be desirable that future $\mathrm{CB}$ generations again incorporate a shortened distal tip.

\section{Limitations of the study}

The current study is a retrospective analysis and is based on a single-center experience in a limited number of patients. Since this is not a randomized controlled trial with proper power calculations any conclusions regarding outcome are hypothesis-generating only. Currently, the CB3 is not available. However, future $\mathrm{CB}$ generations might again incorporate a shorter balloon tip.

\section{Conclusions}

According to available research, this is the first study reporting on procedural characteristics and 1-year clinical outcomes of CB3- as compared to CB2-based PVI. While demonstrating comparable acute and 1-year efficacy, the CB3 provides a significantly increased rate of real-time PV-recordings as compared to CB2 and thus facilitates individual ablation strategies considering individual TTE.

\section{Acknowledgements}

We wish to thank Claudia Kalkowsky, Sanela Dzudzevics, Peter Wohlmuth and Detlef Hennig for their excellent assistance.

Conflict of interest: Christian-Hendrik Heeger received travel grants by St. Jude Medical, Biotronik and Medtronic. Erik Wissner is a consultant to Medtronic. Karl-Heinz Kuck received travel grants and research grants from Biosense Webster, Stereotaxis, Prorhythm, Medtronic, Edwards, Cryocath, and is a consultant to St. Jude Medical, Biosense Webster, Prorhythm, and Stereotaxis. He received speaker's honoraria from Medtronic. Andreas Metzner received speaker's honoraria and travel grants from Medtronic, Biosense Webster 
and Cardiofocus. All other authors have no relevant disclosures.

\section{References}

1. Heeger CH, Wissner E, Knöll M, et al. Three-Year Clinical Outcome After 2nd-Generation Cryoballoon-Based Pulmonary Vein Isolation for the Treatment of Paroxysmal and Persistent Atrial Fibrillation - A 2-Center Experience. Circ J. 2017; 81(7): 974-980, doi: 10.1253/circj.CJ-16-1334, indexed in Pubmed: 28344202.

2. Lemes C, Wissner E, Lin T, et al. One-year clinical outcome after pulmonary vein isolation in persistent atrial fibrillation using the second-generation $28 \mathrm{~mm}$ cryoballoon: a retrospective analysis. Europace. 2016; 18(2): 201-205, doi: 10.1093/europace/euv092, indexed in Pubmed: 25995389.

3. Metzner A, Heeger CH, Wohlmuth P, et al. Two-year outcome after pulmonary vein isolation using the second-generation $28-\mathrm{mm}$ cryoballoon: lessons from the bonus freeze protocol. Clin Res Cardiol. 2016; 105(1): 72-78, doi: 10.1007/s00392-015-0890-8, indexed in Pubmed: 26164844.

4. Metzner A, Reissmann B, Rausch P, et al. One-Year Clinical Outcome After Pulmonary Vein Isolation Using the Second-Generation 28-mm Cryoballoon. Circulation: Arrhythmia and Electrophysiology. 2014; 7(2): 288-292, doi: 10.1161/circep.114.001473.

5. Kuck KH, Brugada J, Fürnkranz A, et al. FIRE AND ICE Investigators. Cryoballoon or Radiofrequency Ablation for Paroxysmal Atrial Fibrillation. N Engl J Med. 2016; 374(23): 2235-2245, doi: 10.1056/NEJMoa1602014, indexed in Pubmed: 27042964.

6. Kirchhof P, Benussi S, Kotecha D, et al. ESC Scientific Document Group . 2016 ESC Guidelines for the management of atrial fibrillation developed in collaboration with EACTS. Eur Heart J. 2016; 37(38): 2893-2962, doi: 10.1093/eurheartj/ehw210, indexed in Pubmed: 27567408.

7. Ciconte G, de Asmundis C, Sieira J, et al. Single 3-minute freeze for second-generation cryoballoon ablation: one-year followup after pulmonary vein isolation. Heart Rhythm. 2015; 12(4): 673-680, doi: 10.1016/j.hrthm.2014.12.026, indexed in Pubmed: 25542427.

8. Heeger $\mathrm{CH}$, Wissner E, Wohlmuth P, et al. Bonus-freeze: benefit or risk? Two-year outcome and procedural comparison of a "bonus-freeze" and "no bonus-freeze" protocol using the second-generation cryoballoon for pulmonary vein isolation. Clin Res Cardiol. 2016; 105(9): 774-782, doi: 10.1007/s00392-0160987-8, indexed in Pubmed: 27085722.

9. Heeger $\mathrm{CH}$, Wissner $\mathrm{E}$, Mathew $\mathrm{S}$, et al. Short tip-big difference? First-in-man experience and procedural efficacy of pulmonary vein isolation using the third-generation cryoballoon. Clin Res Cardiol. 2016; 105(6): 482-488, doi: 10.1007/s00392-015-0944-y, indexed in Pubmed: 26608161.

10. Chierchia GB, Mugnai G, Ströker E, et al. Incidence of real-time recordings of pulmonary vein potentials using the third-generation short-tip cryoballoon. Europace. 2016; 18(8): 1158-1163, doi: 10.1093/europace/euv452, indexed in Pubmed: 26857185.
11. Aryana A, Kowalski M, O’Neill PG, et al. Catheter ablation using the third-generation cryoballoon provides an enhanced ability to assess time to pulmonary vein isolation facilitating the ablation strategy: Short- and long-term results of a multicenter study. Heart Rhythm. 2016; 13(12): 2306-2313, doi: 10.1016/j. hrthm.2016.08.011, indexed in Pubmed: 27503480.

12. Wissner E, Heeger $\mathrm{CH}$, Grahn H, et al. One-year clinical success of a 'no-bonus' freeze protocol using the second-generation $28 \mathrm{~mm}$ cryoballoon for pulmonary vein isolation. Europace. 2015; 17(8): 1236-1240, doi: 10.1093/europace/euv024, indexed in Pubmed: 25868468.

13. Martins RP, Hamon D, Césari O, et al. Safety and efficacy of a second-generation cryoballoon in the ablation of paroxysmal atrial fibrillation. Heart Rhythm. 2014; 11(3): 386-393, doi: 10.1016/j.hrthm.2014.01.002, indexed in Pubmed: 24389575.

14. Fürnkranz A, Bordignon S, Schmidt B, et al. Improved procedural efficacy of pulmonary vein isolation using the novel secondgeneration cryoballoon. J Cardiovasc Electrophysiol. 2013; 24(5): 492-497, doi: 10.1111/jce.12082, indexed in Pubmed: 23398599.

15. Heeger $\mathrm{CH}$, Wissner $\mathrm{E}$, Mathew $\mathrm{S}$, et al. Once Isolated, Always Isolated? Incidence and Characteristics of Pulmonary Vein Reconduction After Second-Generation Cryoballoon-Based Pulmonary Vein Isolation. Circ Arrhythm Electrophysiol. 2015; 8(5): 1088-1094, doi: 10.1161/CIRCEP.115.003007, indexed in Pubmed: 26338833.

16. Reissmann B, Wissner E, Deiss S, et al. First insights into cryoballoon-based pulmonary vein isolation taking the individual timeto-isolation into account. Europace. 2017; 19(10): 1676-1680, doi: 10.1093/europace/euw233, indexed in Pubmed: 28201538.

17. Su W, Kowal R, Kowalski M, et al. Best practice guide for cryoballoon ablation in atrial fibrillation: The compilation experience of more than 3000 procedures. Heart Rhythm. 2015; 12(7): 1658-1666, doi: 10.1016/j.hrthm.2015.03.021, indexed in Pubmed: 25778428 .

18. Metzner A, Burchard A, Wohlmuth P, et al. Increased incidence of esophageal thermal lesions using the second-generation 28-mm cryoballoon. Circ Arrhythm Electrophysiol. 2013; 6(4): 769-775, doi: 10.1161/CIRCEP.113.000228, indexed in Pubmed: 23748208 .

19. Straube F, Dorwarth U, Vogt J, et al. Differences of two cryoballoon generations: insights from the prospective multicentre, multinational FREEZE Cohort Substudy. Europace. 2014; 16(10): 1434-1442, doi: 10.1093/europace/euu162, indexed in Pubmed: 24994074.

20. Rottner L, Fink T, Heeger $\mathrm{CH}$, et al. Is less more? Impact of different ablation protocols on periprocedural complications in second-generation cryoballoon based pulmonary vein isolation. Europace. 2017 [Epub ahead of print], doi: 10.1093/europace/ eux219, indexed in Pubmed: 29016779.

21. Fürnkranz A, Bologna F, Bordignon S, et al. Procedural characteristics of pulmonary vein isolation using the novel thirdgeneration cryoballoon. Europace. 2016; 18(12): 1795-1800, doi: 10.1093/europace/euw012, indexed in Pubmed: 26935947. 